\title{
Report
}

\section{Ultrathin Single-Crystalline Silver Nanowire Arrays Formed in an Ambient Solution Phase}

Byung Hee Hong, Sung Chul Bae, Chi-Wan Lee, Sukmin Jeong, Kwang S. Kim*

National Creative Research Initiative Center for Superfunctional Materials, Department of Chemistry, Division of Molecular and Life Sciences, Pohang University of Science and Technology, Hyojadong, Namgu, Pohang 790-784, Korea.

*To whom correspondence should be addressed. E-mail: kim@ postech.ac.kr

We report the synthesis of single-crystalline silver nanowires of atomic dimensions. The ultrathin silver wires with 0.4 nanometer width grow up to micrometerscale length inside the pores of self-assembled calix[4]hydroquinone nanotubes by electro-/photochemical redox reaction in an ambient aqueous phase. The present sub-nanowires are very stable under ambient air and aqueous environments, unlike previously reported metal wires of $\sim 1$ nanometer diameter which existed only transiently in ultrahigh vacuum. The wires exist as coherently oriented three-dimensional arrays of ultrahigh density, and thus could be employed as model systems for investigating one-dimensional phenomena and as nanoconnectors for designing nanoelectronic devices.

Nanowires have attracted extensive interests in recent years because of their unusual quantum properties and potential use as nanoconnectors and nanoscale devices $(1-3)$. To obtain enhanced physical properties the wires need to be of small diameter, high aspect ratio, and uniformly oriented (2). The stability of the nanowires is also a concern, since metal nanowires of $\sim 1 \mathrm{~nm}$ diameter are reported to have been existed only transiently ( $<10 \mathrm{~s}$ in ultrahigh vacuum) $(1,3)$.

We have recently designed, synthesized, and characterized self-assembled organic nanotube arrays (Fig. 1A) formed by calix[4]hydroquinones (CHQ) (Fig. 1B) in the aqueous phase and found that they form chessboard-like arrays of rectangular pores $(4,5)$. CHQ is an electrochemically-active macrocyclic molecule of calixarene derivative with four hydroquinone (HQ) subunits (6). HQs have been widely used as reducing agents for metallic nano-structures $(7,8)$, in particular, to reduce silver ions to form silver aggregates in acidic solution under photochemical conditions (7), because of the free energy gain through the reduction-oxidation process (9). The inner surface of the pores in the CHQ nanotubes is composed of aromatic $\pi$-faces and $\mathrm{OH}$ groups. In the aqueous phase, partially mobile water molecules are present inside the pores due to hydrophilic $\mathrm{OH}$ groups of CHQs and the water molecules bridging CHQ monomers, and thus help stabilize the silver ions in the pores. In particular, rich $\pi$-electron density of HQ moieties in the CHQ nanotubes enables the CHQ nanotubes to capture metal ions in their pores with high affinity by cation- $\pi$ interactions (10-12). Since the reduced neutral silver atoms do not exhibit strong interactions with the HQ moieties, they readily aggregate into nanowires (Fig. 1, C and D), as the size of the pores closely matches the primitive cell structure of the face-centered cubic (fcc) lattice of the bulk silver.

To prepare the nanowires, the CHQ nanotube powder was soaked in a $1 \mathrm{M} \mathrm{AgNO}_{3}$ aqueous solution for a few minutes, followed by UV irradiation for 30 seconds to accelerate the redox process. The resulting product was filtered and rinsed with distilled water. All the processes were carried out in the solution phase at standard temperature and pressure, whereas most of the previous methods involve extreme conditions like high temperature (13), high pressure (2), or high vacuum (3).

High-resolution electron microscope (HREM) images (14) and the energy dispersive X-ray spectroscopy (EDS) analysis of the silver nanowires in the CHQ nanotube bundles together with the X-ray structure of the CHQ nanotube crystal reveal that single crystalline and monodispersed silver nanowires of $0.4 \mathrm{~nm}$ width are formed inside the pores of the CHQ nanotube arrays (Figs. 2 and 3). The EDS analysis shows that the nanowires are composed of only silver atoms (Fig. 2B). Since the organic elements of the CHQ nanotubes do not exhibit characteristic patterns in HREM imaging at $200 \mathrm{keV}$, the visible patterns (Fig. 2, C through E) emerge solely from the silver atoms. Fig. $2 \mathrm{C}$ shows that the one-dimensional arrays are unidirectional and monodispersed. The uniformity of the HREM image of the nanowires was found up to the length of $\sim 750 \mathrm{~nm}$, indicating the well-ordered onedimensional structure (15). The spots and lines in the HREM image (Fig. 2D) represent the positions of the silver atoms or lattice. The atomic spacing along the wire axis ([110] 
direction) is $0.275 \mathrm{~nm}$ (Fig. 2D), which is $5 \%$ shorter than the corresponding value of the fcc bulk along the [110] direction $(0.289 \mathrm{~nm})$. From the HREM image of the nanowire arrays piled over each other, the periodicity of $1.7 \mathrm{~nm}$ from axis to axis of neighboring wires is observed, as in Fig. 2E where the distance between adjacent black stripes $(1.2 \mathrm{~nm})$ corresponds to the projected view of the pore-to-pore distance of CHQ nanotube arrays on the (110) plane in terms of nanowire array reference basis $(1.7 \mathrm{~nm} / \sqrt{2}=1.2 \mathrm{~nm})(16)$. This indicates the well-ordered three-dimensional arrays of ultrahigh density. Here, it should be noted that two different types of indexing are employed: the silver fcc lattice (in roman fonts) and the nanowire array reference basis (in italic fonts) (Fig. 3, A through C) (17).

The electron diffraction (ED) patterns of the HREM images together with the image simulation show that the silver nanowires are uniformly aligned within the pores of the CHQ nanotube bundles (Fig. 3, D through I). The analysis of the nanowire arrays along the [212] direction (Fig. 3, D through F) confirms existence of single-crystalline silver nanowires. While the one-dimensional structures within carbon nanotubes have been characterized as either individual wires or polydispersed and twisted bundles (18-20), the present silver nanowires samples are observed as coherently oriented arrays which show wire-wire periodicity as well as atomic periodicity. In Fig. 3D, the distance from the origin to the lattice point $3 \overline{4} \bar{l}$ is $3.64 \mathrm{~nm}-1$ corresponding to the reciprocal of the distance between two neighboring silver atoms, while the distance from the origin to the nearest lattice point $\bar{l} 20$ is $1.32 \mathrm{~nm}^{-1}$ corresponding to the reciprocal of the distance between two neighboring wires $(0.76 \mathrm{~nm})$ in Fig. 3E (21). Therefore, the figure exhibits a unique three-

dimensional wire-arrays ED pattern, showing lines of spots in the direction perpendicular to the wire axis (Fig. 3D). This pattern is somewhat similar to the case of MoS2 nanotube arrays (22). We have also observed other intriguing ED pattern images which demonstrate the existence of threedimensional wire-arrays [supplementary material (23)]. The single-crystalline pattern of deformed hexagonal symmetry also matches the calculated pattern with the zone axis [453] with an error of only $2 \%$ (Fig. 3, G through I). The low-index orientation of the nanowire arrays exhibits pseudo bulk-like patterns (Fig. 3, $\mathrm{G}$ and $\mathrm{H}$ ) which could not be observed from any orientations of the bulk (fcc) silver crystal (21). Fig. 3G obtained from a small section of the clear one-dimensional wire-arrays shown in low-magnification image (Fig. 3J) supports that the deformed hexagonal pattern originates from the array architectures. In addition, the HREM images do not show the surface reconstruction observed in the gold nanobridge (24) and nanorod (25). Thus, all HREM images support that the silver structures exist as nanowires, but not bulk or electro-plated surfaces. In particular, Figs. 2D, 3E, and $3 \mathrm{H}$ demonstrate the uniformly oriented three-dimensional arrays with coherent orientations of silver atoms in neighboring wires (26).

Geometrical structures of the nanowires are available from HREM, which we compare them with theoretical results, and for which we investigate the electronic structure. We have carried out density functional electronic structure calculations (27), using the ultrasoft pseudopotential plane wave method (28). A supercell was used so that the neighboring nanowires are sufficiently separated. The calculated atomic layer spacing along the [110] wire axis $(0.278 \mathrm{~nm})$ is $4 \%$ smaller than the corresponding calculated bulk value $(0.289 \mathrm{~nm})$, which is in good agreement with the HREM result $(0.275$ $\mathrm{nm})$. The interatomic spacing along the [001] direction is determined to be $0.395 \mathrm{~nm}$, which is $3 \%$ smaller than the corresponding bulk value $(0.409 \mathrm{~nm})$. A slightly more significant compression of the inter-atomic distances is observed across the cross section rather than along the wire axis. This is due to increased interactions between the inner and surface atoms caused by the high electron density near the surfaces in the case of metals, which has also been observed in silver nanoclusters (29). The calculated electronic structure shown in Fig. 4 suggests that the silver nanowire is metallic (30). The s bands cross the Fermi level at three points, suggesting the existence of three conducting channels for electronic transport as a quantum wire. On the other hand, the calculation predicts that the CHQ nanotube is an insulator (with $\sim 3 \mathrm{eV}$ band gap) which shields the conduction between nanowires.

Since the silver nanowires are confined and protected by the organic supporter, the shielding effect prevents the silver nanowires from oxidation. In addition, the organic supporter also provides long term stability for the ultrathin linear nanowires which would otherwise only show transient stability. In the samples stored under ambient air or aqueous environments for more than a month, no notable changes have been observed in the analysis of FTIR spectra and HREM images. Finally, considering the oxidation potential of the HQ moieties in the CHQ and the reduction potentials of metal ions, it should be possible to prepare nanowires with other metallic elements such as gold, palladium, platinum, and mercury in a similar way.

\section{References and Notes}


1. A. I. Yanson, G. R. Bollinger, H. E. van der Brom, N. Agraït, J. M. van Ruitenbeek, Nature 395, 783 (1998).

2. J. D. Holmes, K. P. Johnston, R. C. Doty, B. A. Korgel, Science 287, 1471 (2000).

3. Y. Kondo, K. Takayanagi, Science 289, 606 (2000).

4. B. H. Hong, J. Y. Lee, C.-W. Lee, J. C. Kim, S. C. Bae, K. S. Kim, J. Am. Chem. Soc. (accepted).

5. Under ambient conditions, $0.1 \mathrm{~g}$ of $\mathrm{CHQ}$ monomer dissolved in $50 \mathrm{ml}$ of 1:1 water-acetone solution, which leads to the formation of nanotube bundles as the acetone dries up. In the presence of cesium sulfate, the bundles grow into needle-like dark brown crystals. The X-ray structure of the crystal has been deposited in the Cambridge Crystallographic Data Centre as supplementary publication no. CCDC-161593. The inner surface of the CHQ nanotube is composed of hydroquinone (HQ) moieties framed by four one-dimensional $\mathrm{H}$-bond arrays formed with $\mathrm{OH}$ groups of quinones and water molecules. The nanotube bundles are formed by $\pi-\pi$ stacking interactions between HQ moieties of nanotubes. The simple view of inner surface can be seen in Fig. 1D and the supplementary material (23), and the more detailed views of the molecular structure around the pores as well as the details of X-ray and IR characterization of the CHQ nanotubes are available in (4).

6. M. Gómez-Kaifer, P. A. Reddy, C. D. Gutsche, L. Echegoyen, J. Am. Chem. Soc. 116, 3580 (1994).

7. E. Braun, Y. Eichen, U. Sivan, G. Ben-Yoseph, Nature 391, 775 (1998).

8. T. A. Taton, C. A., Mirkin, R. L. Letsinger, Science 289, 1757 (2000).

9. Once the silver cations are captured inside the pores, the redox process between $\mathrm{Ag}^{+}$and $\mathrm{CHQ}$ takes place spontaneously with the positive reduction potential $\left(\mathrm{E}^{\mathrm{o}}=\right.$ $0.1 \mathrm{~V}): \mathrm{Ag}^{+}+\mathrm{e}^{-} \rightarrow \mathrm{Ag}\left(\mathrm{E}^{\mathrm{o}}=0.8 \mathrm{~V}\right)$ and $\mathrm{Q}+2 \mathrm{H}^{+}+2 \mathrm{e}^{-} \rightarrow$ $\mathrm{HQ}\left(\mathrm{E}^{0}=0.7 \mathrm{~V}\right)$, where $\mathrm{Q}$ is the quinone moiety. The number of $\mathrm{Ag}$ atoms is half of maximum reduction sites of the CHQ nanotubes.

10. D. A. Dougherty, Science 271, 163 (1996).

11. H. S. Choi, S. B. Suh, S. J. Cho, K. S. Kim, Proc. Natl. Acad. Sci. U.S.A. 95, 12094 (1998).

12. K. S. Kim, P. Tarakeshwar, J. Y. Lee, Chem. Rev. 100, 4145 (2000).

13. M. P. Zach, K. H. Ng, R. M. Penner, Science 290, 2120 (2000).

14. The HREM images were obtained using $200 \mathrm{keV} \mathrm{JEOL}$ JEM-2010F.

15. More extensive versions of the electron microscope images are available in the supplementary material (23).
16. We have also observed the HREM images of the stripeto-stripe distance of $\sim 0.7 \mathrm{~nm}$ corresponding to the projected wire-wire distance on the (210) plane [supplementary material (23)].

17. In the nanowire array reference basis, it should be noted that the distance from the origin to the [001] direction $(0.275 \mathrm{~nm})$ is much shorter than those from the origin to the [100] and [010] directions $(1.7 \mathrm{~nm})$.

18. R. M. Meyer et al., Science 289, 1325 (2000).

19. D. Ugarte, A. Chatelain, W. A. de Heer, Science 274, 1897 (1996).

20. L. Henrard, A. Loiseau, C. Journet, P. Bernier, Eur. Phys. J. 13, 661 (2000).

21. In Fig. 3E, the projected distance between two neighboring on the (212) plane is $0.76 \mathrm{~nm}\left[=1.7 \mathrm{~nm} /\left(2^{2}+\right.\right.$ $\left.\left.1^{2}\right)^{1 / 2}\right]$. In Fig. 3H, that on the (453) plane is $0.265 \mathrm{~nm}$ [= $\left.1.7 \mathrm{~nm} /\left(4^{2}+5^{2}\right)^{1 / 2}\right]$. The distance from the origin to the lattice point $\overline{5} 40$ is $3.77 \mathrm{~nm}^{-1}$ corresponding to the reciprocal distance between two neighboring wires [ $(0.265$ $\left.\mathrm{nm})^{-1}\right]$. This is contrasted to the reciprocal distance $[(0.236$ $\mathrm{nm})^{-1}=4.24 \mathrm{~nm}^{-1}$ ] observed in the bulk silver along the [110] direction.

22. M. Remskar et al., Science 292, 479 (2001).

23. Supplementary Web material is available on Science Online at www.sciencemag.org/cgi/content/full/1062126/DC1.

24. Y. Kondo, K. Takayanagi, Phys. Rev. Lett. 79, 3455 (1997)

25. Z. L. Wang, R. P. Gao, B. Nikoobakht, M. A. El-Sayed, J. Phys. Chem. B 104, 5417 (2000).

26. The silver nanowires which have the fcc primitive cell structure, are aligned with uniform orientations (Fig. 3B). In the case of CHQ nanotubes, the $\mathrm{O}$ atoms of water molecules bridging neighboring CHQs are nearest to the pore axis $(\sim 0.28 \mathrm{~nm})$, while atoms of CHQs are somewhat far from the pore axis $(\sim 0.4 \mathrm{~nm})$. Once inside the pores and reduced, the silver atoms interact with the non-mobile bridging water molecules leading to the coherent orientation of silver atoms in neighboring nanowires. Although some of the H-bonding structure in the pore is changed by the formation of the silver wire, the overall skeletal structure of the nanotube arrays is determined by the $\pi-\pi$ stacking. Thus, the overall structure of the CHQ tube arrays with silver nanowires is not significantly different from that of the pure CHQ nanotube arrays. This is evidenced from experiments that the pore-to-pore distance in the X-ray structure of the nanotube arrays and the wire-to-wire distance in the HREM structure of the 
nanowire arrays inside the nanotube bundles are practically the same. We have also seen many other HREM patterns that demonstrate well-oriented nanowires form in the pores of the CHQ nanotube arrays [a few of them are in the supplementary material (23)].

27. D. M. Ceperley, B. J. Alder, Phys. Rev. Lett. 45, 566 (1980).

28. D. Vanderbilt, Phys. Rev. B 41, 7892 (1990).

29. J. Yoon, K. S. Kim, K. K. Baeck, J. Chem. Phys. 112, 9335 (2000).

30. We have investigated the electron energy loss spectroscopy (EELS) in HREM for both the bulk and nanowires [supplementary material (23)]. In the nanowires, the volume plasmon peak at $7.4 \mathrm{eV}$ corresponding to the bulk almost disappears, but a weak peak at $\sim 6 \mathrm{eV}$ seems to appear though it is not clearly resolved due to the strong zero-loss peak. Furthermore, when the samples are exposed to the electron beam of HREM, we find that the silver wires in the supports are stable. This is possibly due to high conductivity, as less conductive palladium wires (which have a higher boiling point) tend to evaporate due to local heating. This supports the idea that the silver wires are conductive. The contact problem needs to be solved for the direct measurement of the conductivity.

31. This research was supported by KISTEP(CRI) and partly by BK21. We thank C. G. Park, S. G. Hong, K. W. Cho, B. I. Kang, B. W. Seo (POSTECH), G. S. Park, M. Kim (SAIT), and J. C. Park (Hynix Co.) for HREM, FE-SEM, and EELS analyses, and S. J. Youn for calculations. We also thank S. Granick (U. Illinois), H. J. Lee, S. Lee, and J. W. Jung (POSTECH) for discussion.

1 May 2001; accepted 27 August 2001

Published online 6 September 2001;

10.1126/science. 1062126

Include this information when citing this paper.

Fig. 1. Organic nanotube templates and a silver nanowire inside the nanotube. (A) A schematic view of CHQ nanotube arrays based on the X-ray analysis (4) of the crystal (orthorhombic, space group $\mathrm{C}_{\mathrm{mmm}}$ with lattice constants: $a=$ $2.50 \mathrm{~nm}, b=2.33 \mathrm{~nm}, c=1.16 \mathrm{~nm}$ and $\alpha=\beta=\gamma=90^{\circ}$ ) reveals that the pores of $0.6 \times 0.6 \mathrm{~nm}^{2}$ (with van der Waals volume excluded) are separated by $1.7 \mathrm{~nm}$ from the neighboring ones. Water molecules are not shown for clarity. $\mathrm{O}$ atoms are shown in red. (B) CHQ monomer. (C and D) Top and side views of a silver nanowire inside a CHQ nanotube template, respectively. The solvent-accessible surface model in (C) and the stick model in (D) show schematic views of the CHQ nanotubes. Red color of the surface represents the negative electrostatic potential on oxygen atoms. The space-filled models (blue) represent the silver nanowires.

Fig. 2. HREM images and EDS elemental analysis of the nanowires. (A) Low-magnification image of the silver nanowires formed in CHQ nanotube templates (scale bar, $100 \mathrm{~nm}$ ). (B) EDS elemental analysis indicating that the only metal composition is silver. $\mathrm{L}_{\alpha 1}$ and $\mathrm{L}_{\beta 1}$ are the transitions responsible for the $\mathrm{X}$-ray fluorescence lines of silver. $(\mathbf{C})$ Magnified image of (A) showing that one-dimensional structures are unidirectional and monodispersed (scale bar, 2 $\mathrm{nm}$ ). (D) Magnified image of (C) in atomic resolution showing coherent orientations of silver atoms in different wires. The white arrow indicates the wire direction, [110] (scale bar, $1 \mathrm{~nm}$ ). (E) Image of the nanowire arrays piled over each other (scale bar, $2 \mathrm{~nm}$ ).

Fig. 3. Structural analysis of the single-crystalline primitive silver nanowire arrays. (A) Three-dimensional model of the 4 $\times 4$ silver nanowire arrays. $z$ axis corresponds to [110] direction of fcc lattice. (B) Zone axis notations (italic fonts) in terms of the nanowire array reference basis. The [100], [010], and [001] directions in this wire array reference basis correspond to [001], [ $\overline{1} 10]$, and [110] of the fcc lattice, respectively. (C) Top and side views of a primitive silver nanowire model. (D) Selected area diffraction pattern for zone [212] (scale bar, $2 \mathrm{~nm}^{-1}$ ) and simulated pattern (inset). (E) HREM image (scale bar, $1 \mathrm{~nm}$ ) and simulated image (inset; sample thickness $=17 \mathrm{~nm}$, defocus $=70 \mathrm{~nm}$ ) corresponding to (D). (F) Nanowire array orientation corresponding to $(\mathrm{E})$. (G) Nano-beam diffraction pattern for zone [453] (scale bar, $5 \mathrm{~nm}^{-1}$ ). (H) HREM image (scale bar, 1 $\mathrm{nm}$ ) and simulated image (inset; sample thickness $=17 \mathrm{~nm}$, defocus $=80 \mathrm{~nm}$ ) corresponding to $(G)$. (I) Nanowire array orientation corresponding to $(\mathrm{H})$. (J) HREM image of a nanotube bundle with nanowires inside the pores (scale bar, $10 \mathrm{~nm})$. Note that image $(\mathrm{H})$ is the highly magnified HREM image of the dotted square.

Fig. 4. Electronic band structure (A) and density of states (DOS) (B) of the single-crystalline primitive silver nanowire along the [110] direction. 


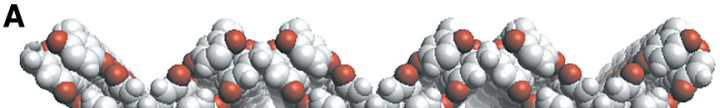

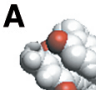
(1)

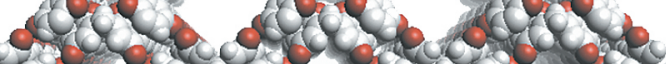

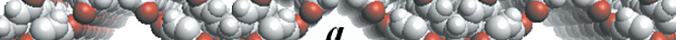

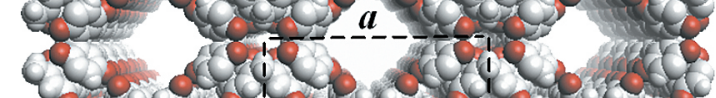
4 -

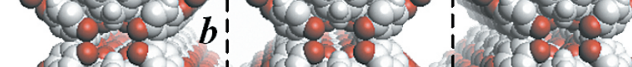

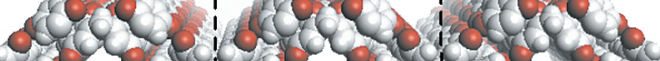

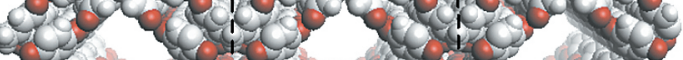

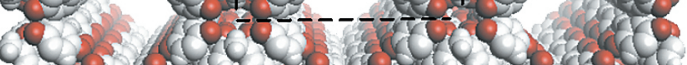

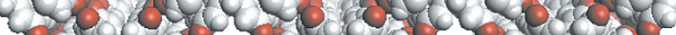

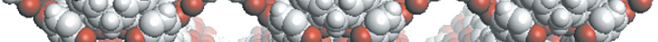

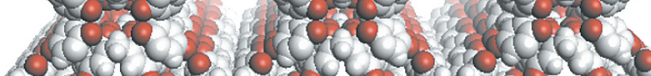

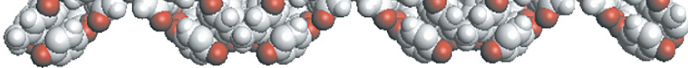

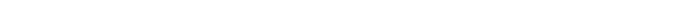

$c{ }_{-3}^{-3}$
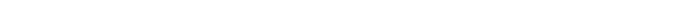
A 蜜密 B

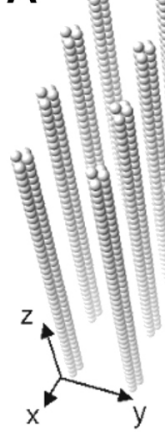

S

\section{D}

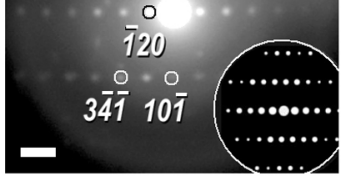

C

G.

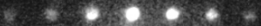

s. O2,

- 250.040

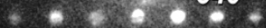

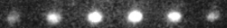

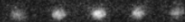

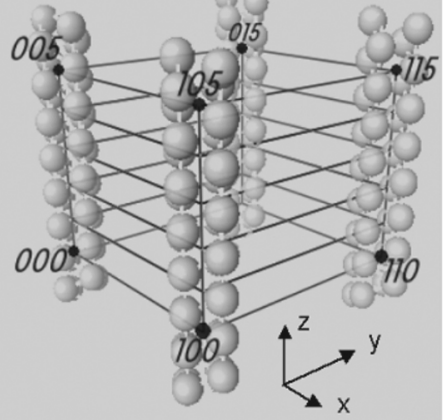

C

$(\underbrace{\infty}_{[110]} z$

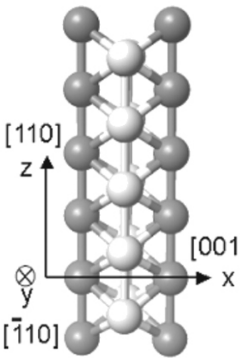

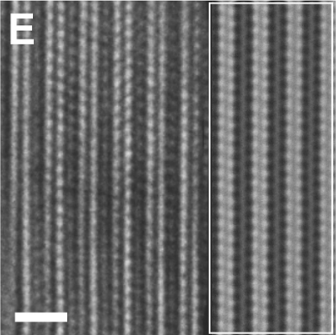

$F$

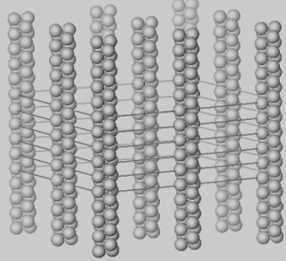

H.

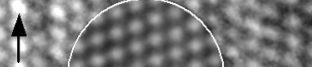

110

110

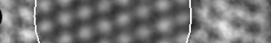

in $\underbrace{1}_{-1}$

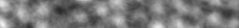

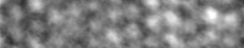

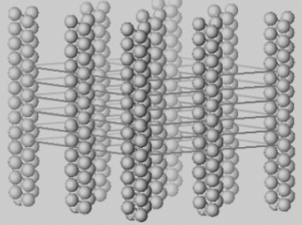




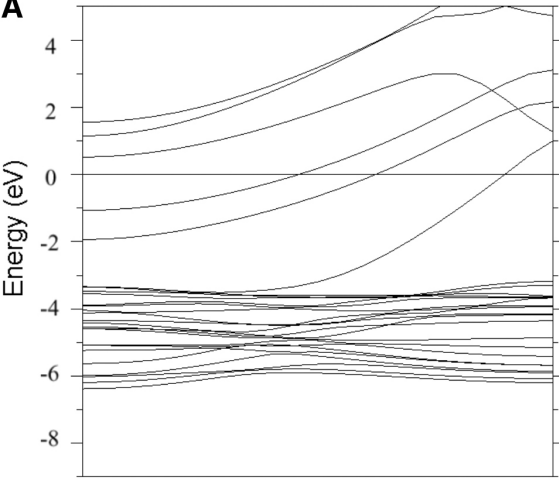

$\Gamma$

$B$
$B$
0
0
0
0
0

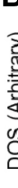

500

중 400

300

200
100

0

$-10$

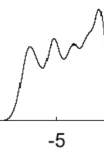

Energy $(\mathrm{eV})$ 